\title{
Machine learning with asymmetric abstention for biomedical decision-making
}

\author{
Mariem Gandouz ${ }^{1}$, Hajo Holzmann² and Dominik Heider ${ }^{1 *}$
}

\begin{abstract}
Machine learning and artificial intelligence have entered biomedical decision-making for diagnostics, prognostics, or therapy recommendations. However, these methods need to be interpreted with care because of the severe consequences for patients. In contrast to human decision-making, computational models typically make a decision also with low confidence. Machine learning with abstention better reflects human decision-making by introducing a reject option for samples with low confidence. The abstention intervals are typically symmetric intervals around the decision boundary. In the current study, we use asymmetric abstention intervals, which we demonstrate to be better suited for biomedical data that is typically highly imbalanced. We evaluate symmetric and asymmetric abstention on three real-world biomedical datasets and show that both approaches can significantly improve classification performance. However, asymmetric abstention rejects as many or fewer samples compared to symmetric abstention and thus, should be used in imbalanced data.
\end{abstract}

Keywords: Medical data science, Machine learning, Classification, Diagnostics

\section{Introduction}

Machine learning (ML) and artificial intelligence (AI) have entered many areas of life and will also pave the way for a new era in biomedicine. These methods can improve medical treatment or diagnosis, identify novel subtypes, or provide new insights into survival prognostics. These methods consider all facets of data types, e.g., clinical health records, images, or omics data. Biomedical decision support systems based on ML and AI have entered many different studies and biomedical fields, e.g., Oncology [4], Pathology [10, 21, 35], Diabetes [6, 27], Human Genetics [20], and Infectious Diseases [14, 19, 28] as part of a growing trend towards precision medicine.

Overall, there is great potential for biomedical decision support systems based on ML and AI techniques and they have become key players in disease diagnostics, prognostics, and therapeutics [34]. However, biomedical

\footnotetext{
*Correspondence: dominik.heider@uni-marburg.de

${ }^{1}$ Department of Data Science in Biomedicine, Faculty of Mathematics

and Computer Science, University of Marburg, 35032 Marburg, Germany

Full list of author information is available at the end of the article
}

datasets have very specific characteristics and suffer from many caveats regarding ML and AI. They often have a small number of samples and many parameters, a phenomenon called the small- $n$-large- $p$ problem, missing values, and typically high class imbalance. Furthermore, biomedical decision support systems need to be probabilistically interpretable, typically addressed by calibration methods $[1,26]$. While small- $n$-large- $p$ and missing values are addressed by feature selection (also called biomarker discovery) approaches [23] and imputation techniques [29], the class imbalance is typically addressed by either down-sampling or data augmentation techniques. Moreover, uncertainty is critical when it comes to a medical decision. In contrast to human decision making, computational models typically make a decision also with low confidence. Machine learning with abstention better reflects human decision-making by introducing a reject option for samples with low confidence. The abstention intervals are typically symmetric intervals around the decision boundary. Thus uncertain predictions, i.e., predictions with low confidence or close to the decision

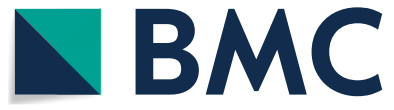

(c) The Author(s) 2021. Open Access This article is licensed under a Creative Commons Attribution 4.0 International License, which permits use, sharing, adaptation, distribution and reproduction in any medium or format, as long as you give appropriate credit to the original author(s) and the source, provide a link to the Creative Commons licence, and indicate if changes were made. The images or other third party material in this article are included in the article's Creative Commons licence, unless indicated otherwise in a credit line to the material. If material is not included in the article's Creative Commons licence and your intended use is not permitted by statutory regulation or exceeds the permitted use, you will need to obtain permission directly from the copyright holder. To view a copy of this licence, visit http://creativecommons.org/licenses/by/4.0/. The Creative Commons Public Domain Dedication waiver (http://creativeco mmons.org/publicdomain/zero/1.0/) applies to the data made available in this article, unless otherwise stated in a credit line to the data. 
boundary, are abstained when the consequences of the wrong classification can be severe, e.g., wrong treatment of a patient [15]. A symmetric abstention interval can be defined based on the prediction scores for biomedical decision support systems based on binary classification models. It is considered to be a range of test scores that is uncertain and does not necessitate a decision, and the test results are trichotomized into positive, negative, and undecided diagnoses.

Classifiers with abstention were first introduced by Chow [9] and further developed by Tortorella [30, 31]. Chow [9] derived a general error and reject trade-off relation for the Bayes optimum recognition system requiring the assumption of complete knowledge of the a priori probability distribution of the classes and the posterior probabilities (for instance, the distributions of the test results to be normal in both healthy and diseased subjects), which are usually not completely known in real-world problems. Thus, the reliance of this method on several assumptions represents an important limitation. Fumera et al. [12] demonstrated that Chow's rule does not perform well if the a posteriori probabilities are affected by errors, suggesting the use of multiple reject thresholds, one for each class. The threshold is placed on the maximum a posteriori probability similar to Chow's rule [8]). However, each class has a different threshold. Their results using nearest neighbor and neural network classifiers show that this approach outperforms the parametric assumption. Herbei and Wegkamp [15] developed excess risk bounds for the classification with a reject option setting where the loss function is the $0-1$ loss, extended such that the cost of each reject point is $0 \leq d \leq 1 / 2$ (cost model). This approach generalizes the excess risk bounds of Tsybakov [32] for standard binary classification without rejection (which is equivalent to the case $d=1 / 2$ ). This approach is further extended by Bartlett and Wegkamp [3] in various ways, including the use of the hinge loss function for efficient optimization. Nguyen et al. [24] developed an approach for abstention in multiclass problems based on pairwise comparison and integer programming, and separated epistemic, i.e., uncertainty caused by lack of information, and aleatoric uncertainty, i.e., due to intrinsic randomness. Very recently, Mortier et al. [22] developed a framework for Bayes-optimal prediction in multi-class problems, i.e., the subset of class labels with the highest expected utility. Campagner et al. [5] proposed a three-way-in and three-way-out approach, which is based on partially labeled data and abstention. They analyzed to what extent a classifier can make reliable prediction based on uncertain biomedical data.

While abstention intervals are typically considered to be symmetric, the goal of the current study is to show that asymmetric intervals are better suited for biomedical data, as these datasets are often imbalanced. We propose a simple, efficient, and novel method to optimally build an asymmetric type of abstaining binary classifiers using an asymmetric abstention interval around the intersection between the two distributions of positive samples (i.e., cases) and negative samples (controls) based on Pareto optimization, similar to the approach proposed by Herbei and Wegkamp [15].

\section{Methods}

As a starting point, such a standard should satisfy the following requirements: (1) include information on the population providing the training data, in terms of data sources, cohort selection; (2) include training data demographics in a way that enables a comparison with the population the model is applied to; (3) provide detailed information about the model architecture and development so as to interpret the intent of the model and compare it to similar models and permit replication; and (4) transparently report model evaluation, optimization, and validation to clarify how local model optimization can be achieved and enable replication and resource sharing

\section{Data}

We used three real-world biomedical datasets in our study covering different diseases/scenarios from different fields, namely oncology and reproduction. These datasets address breast cancer, prostate cancer, and cardiotocography to reflect different sample sizes and class imbalances. The datasets were collected from the UCI Machine Learning Repository [11]. The smallest dataset has 72 samples, and the largest dataset consists of 1831 samples. On average, the datasets have 540 samples, the median is 426 , first and third quartiles are 124.5 and 713 , respectively. The imbalance differs between 2.23 and $37.26 \%$ concerning the cases (i.e., positive class). On average, the imbalance is $18.42 \%$ (median is $17.7 \%$ ), with the first and third quartiles at $9.78 \%$ and $28.49 \%$, respectively. The number of features ranges from 3 to 32, on average 15 (median is 11), with the first and third quartiles of 9 and 21, respectively.

An overview of the datasets can be found in Table 1. We removed all samples and features with missing values. Thus the numbers may differ slightly from the original number of samples and features.

The Breast Cancer diagnostics dataset (wdbc) consists of 569 breast cancer patients [357 benign, 212 (37.3\%) malignant] with 30 attributes. Features are computed from a digitized image of a fine needle aspirate (FNA) of a breast mass. They describe characteristics of the cell nuclei present in the image. All cancers and some of the benign masses were histologically confirmed. Cancer patients were given standard surgical and chemotherapy treatment. Adjunctive radiotherapy was given when indicated [33]. No missing attribute values. 
Table 1 Overview of the datasets

\begin{tabular}{llllll}
\hline Name & Samples & Cases & Controls & Percentage & Features \\
\hline wdbc & 569 & 212 & 357 & 37.3 & 30 \\
pc & 376 & 225 & 151 & 59.8 & 9 \\
ctg & 1831 & 176 & 1655 & 9.6 & 22 \\
\hline
\end{tabular}

Percentage represents the percentage of positive samples (i.e., cases) in the dataset. Features refers to the number of independent variables / features in the dataset

The Prostate Cancer (pc) dataset consists of 376 samples [225 (59.8\%) cancer, 151 (40.2\%) controls] with nine features, e.g., age, race, and several clinical parameters [17].

The Cardiotocography dataset (ctg) consists of 1,831 fetal cardiotocograms, of which 1,655 are normal, and 176 have been classified as pathologic (9.6\%). The dataset provides 22 features that have been calculated based on the cardiotocograms [2].

In Table 2, we report the data according to the MINIMAR standard to improve reproducibility [16].

\section{Implementation}

All analyses have been carried out in Python v.3.8.5 with pandas (v.1.1.3), seaborn (v.0.11.0), Matplotlib (v.3.3.2), NumPy (v.1.19.2), scikit-learn (v.0.23.2), and Plotly (v.4.14.2).

\section{Machine learning}

In supervised ML, the data is given by a training set

$$
\mathcal{T}=\left\{\left(\mathbf{x}_{1}, y_{1}\right), \ldots,\left(\mathbf{x}_{m}, y_{m}\right)\right\} \subset(\mathcal{X} \times \mathcal{Y})^{m},
$$

with $m$ data pairs $\left(\mathbf{x}_{\mathbf{i}}, y_{i}\right)$, where $\mathbf{x}_{\mathbf{i}}$ is a vector of the observations for the $i$ th data point and $y_{i}$ is its class label. The elements of the training set are called training data. $\mathcal{X}$ is called the feature space and the dimension $n$ of this space corresponds to the number of features $X_{1}, \ldots, X_{n}$, which are used to describe the observations. Hence $\mathbf{x}_{i}=\left(x_{i 1}, \ldots, x_{i n}\right)^{\top} \in \mathcal{X}$ for $i=1, \ldots, m$, where $x_{i j}$ is the value of the feature $X_{j}(j=1, \ldots, N)$ in the $i$ th observation. Furthermore, each observation $\mathbf{x}_{\mathbf{i}}$ is associated with a class label $y_{i} \in \mathcal{Y}$, where $\mathcal{Y}$ denotes the set of possible labels (sample space for short). In the simplest case, the binary classification, the set $\mathcal{Y}$ consists of only two class labels, which are usually referred to as positive (cases) and negative (controls); or +1 and 0 .

The goal of supervised learning is thus to learn the relationships between the observations/features and the class label, based on the training set, to assign a class label to an observation as accurately as possible. Given the training set, the ML method learns a decision function (also called a classifier or model)

$$
f: \mathcal{X} \rightarrow \mathcal{Y}
$$

that performs classifications by mapping the observations from the feature space $\mathcal{X}$ to the sample space $\mathcal{Y}$ and reducing the error rate iteratively.

However, the decisions made by the model may be wrong for some instances, mainly when these instances are close to the binary decision border.

Thus, we extend the definition of a standard classifier, and we add a new label ${ }^{\circledR}$, which is referred to as abstaining: Given $\mathcal{X}$ and $\mathcal{Y}$ as defined above, an abstaining classifier is defined as a classifier which labels an instance $\mathbf{x}_{\mathbf{i}} \in \mathcal{X}$ with an element from $\mathcal{Y} \cup\left\{{ }^{\oplus}\right\}$.

In order to evaluate the performance of symmetric abstention and asymmetric abstention, we analyzed two scenarios, namely (1) the imbalanced data as it is and (2) down-sampled, balanced data.

To compare the different scenarios, we used the Logistic Regression (LR) as a base model. As the abstention procedure is independent of the ML method used, these results can be generalized for other ML models.

Table 2 Data sets description

\begin{tabular}{llll}
\hline \multicolumn{2}{l}{ Study population and setting } & & \\
\hline Name & Population & Study setting & Data source \\
\hline wdbc & Breast cancer patients & U.S. hospital & Digitized images \\
pc & Prostate cancer patients & U.S. hospital & Adults \\
ctg & Pregnant women & E.U. hospital & Fetal cardiotocograms \\
\hline Patient demographic characteristics & & Unborn \\
\hline Name & Age & Sex & Race \\
\hline wdbc & Not provided & $100 \%$ female & Ethnicity \\
pc & mean $66.04 y$ & $100 \%$ male & No.45\% white, 9.55\% black \\
$c t g$ & Not provided & Not provided & Not provided \\
\hline
\end{tabular}




\section{Statistical evaluation}

The LR models were trained and evaluated based on stratified hold-out validation and split into training and test data. We used $40 \%$ of the data as training data for the wdbc and pc datasets and 50\% for training with the ctg dataset. We used the Matthews correlation coefficient (MCC)

$$
\mathrm{MCC}=\frac{\mathrm{TP} \cdot \mathrm{TN}-\mathrm{FP} \cdot \mathrm{FN}}{\sqrt{(\mathrm{TP}+\mathrm{FP}) \cdot(\mathrm{TP}+\mathrm{FN}) \cdot(\mathrm{TN}+\mathrm{FP}) \cdot(\mathrm{TN}+\mathrm{FN})}}
$$

with true positives (TP), true negatives (TN), false positives (TP) and false negatives (FN) to estimate the performance of the models as this metric has been shown to be particularly well-suited for imbalanced data [7].

In Tables 3 and 4, we report the model architecture and evaluation description according to the MINIMAR standard to improve reproducibility [16].

\section{Symmetric and asymmetric abstention}

The distributions of the test scores of controls (negative class) and cases (positive class) typically overlap in realworld scenarios, and as a result, there are both errors and correct decisions for the test scores between an upper $(\mathrm{U})$ and lower (L) bounds within this range of overlap. In order to find the best symmetric and asymmetric abstention intervals for the test scores, i.e., the intervals that reduce wrong classifications but at the same time can classify most of the data, we used the maximum product of the MCC and the number of classified samples, i.e., samples outside the abstention interval.

\section{Symmetric abstention}

The upper and lower bounds of the symmetric abstention interval are defined with both curves as preliminary cut-point. The default is to use a threshold of 0.5 as the cut-off. Let us assume that $\Delta$ is the best width of the symmetric abstention interval. Thus, the symmetric abstention interval method will output $\Delta / 2$ representing best interval. This means the best symmetric abstention interval is straightforward, simply requiring computing $[L, U]$ with $L=0.5$ - best interval and $U=0.5+$ best interval.

Furthermore, it is essential to note that the binary classifier needs to be already trained and give us the classes' probabilities. Probabilistic interpretation is, for instance, possible for the logistic regression but may not be directly possible for other ML methods, e.g., deep neural networks or support vector machines. In order to provide probabilities for any ML model, calibration methods need to be employed [26].

The main functions for the symmetric abstention are fit () and cost(). fit () finds the value of the best interval. It takes test features (X_test), test labels $\left(y \_t e s t\right)$, abstention interval minimum width (low), abstention interval maximum width (high), and the distance between two adjacent values at each incremental abstention level (step) as input. We set the parameters for the grid search as follows: low $=0$, high $=0.16$, and step $=0.01$. Starting at 0 , which means no abstention interval at all and then going as high as 0.16 . The values represent probabilities, thus, the maximum value can be 0.5 (i.e., 0.5 abstention at each side covers the whole probability range from 0 to 1 ). Max is set to 0.16 in order to stop at 0.15 taking a $1 \%$ abstention margin, which covers basically $2 \%$ as it goes from the left side and the right side of the threshold, and then 0.02, 0.03, etc. up to 0.15 , which covers $30 \%$ of the range.

Next, the intervals are initialized the MCC and the fractional size of the samples (i.e., between 0 and 1; 0 corresponds to no data at all left, and 1 corresponds to all the data) are calculated. Each time the symmetric abstention interval grows, the size of the samples will decrease. Our two conflicting goals are maximizing the MCC while maximizing the size of the testing set that will be classified. We consider this problem as a Pareto optimization problem (also known as multi-objective optimization), where no single solution exists that optimizes each objective simultaneously, i.e., a problem for which there are many possible

Table 3 Architecture description

Model architecture

\begin{tabular}{llll}
\hline User & Task & Architecture & Features \\
\hline Clinicians & Prediction & Logistic regression & Documented and provided for all models in detail \\
\hline
\end{tabular}

Table 4 Evaluation description

Model evaluation

\begin{tabular}{llll}
\hline Optimization & Internal validation & External validation & Transparency \\
\hline Documented and provided for all models in detail & Stratified hold-out validation & Not performed & Data publicly available, code on request \\
\hline
\end{tabular}


solutions from amongst which we want to find "the best". Obviously, if we maximize for mcc_values, we cannot maximize for sample sizes and vice versa. After that, a possibly infinite number of Pareto optimal solutions are found. To overcome such problems and choose, we have to add additional subjective preference information and find a single solution that satisfies it. If no additional subjective preference information can be made, all Pareto optimal solutions are considered equally good. Thus, a set of Pareto optimal solutions will be generated and the best one can be selected according to the additional subjective preference as being the score $=m c c$ values $*$ sizes. We choose one of the obtained solutions using a simple one-dimensional grid search approach for function optimization.

The cost () function returns the MCC and the fractional size of the testing set. It takes as input the test features
( $X \_$test), test labels $\left(y \_\right.$test), and the chosen interval (inter$v a l$ ), which is initialized with 0 . After a sanity check, i.e., that interval $\in\{0,0.5\}$, the class probabilities of the test features are predicted and obtain the maximum probability for the samples. Any prediction that is not in the suitable range, which is $\{$ maximum probability -0.5$\}$ (that is to center it), will be eliminated. All predictions that have an absolute value smaller than the interval are removed. All predictions with values larger than the interval are kept. Next, the size of the remaining samples is calculated, i.e., the test features and test labels that are outside the interval. These samples are predicted with the model, i.e., they are outside the abstention interval to obtain $M C C$ and the corresponding number of samples (fractional size). The pseudocode for the symmetric abstention optimizer algorithm is shown in algorithm 1 .

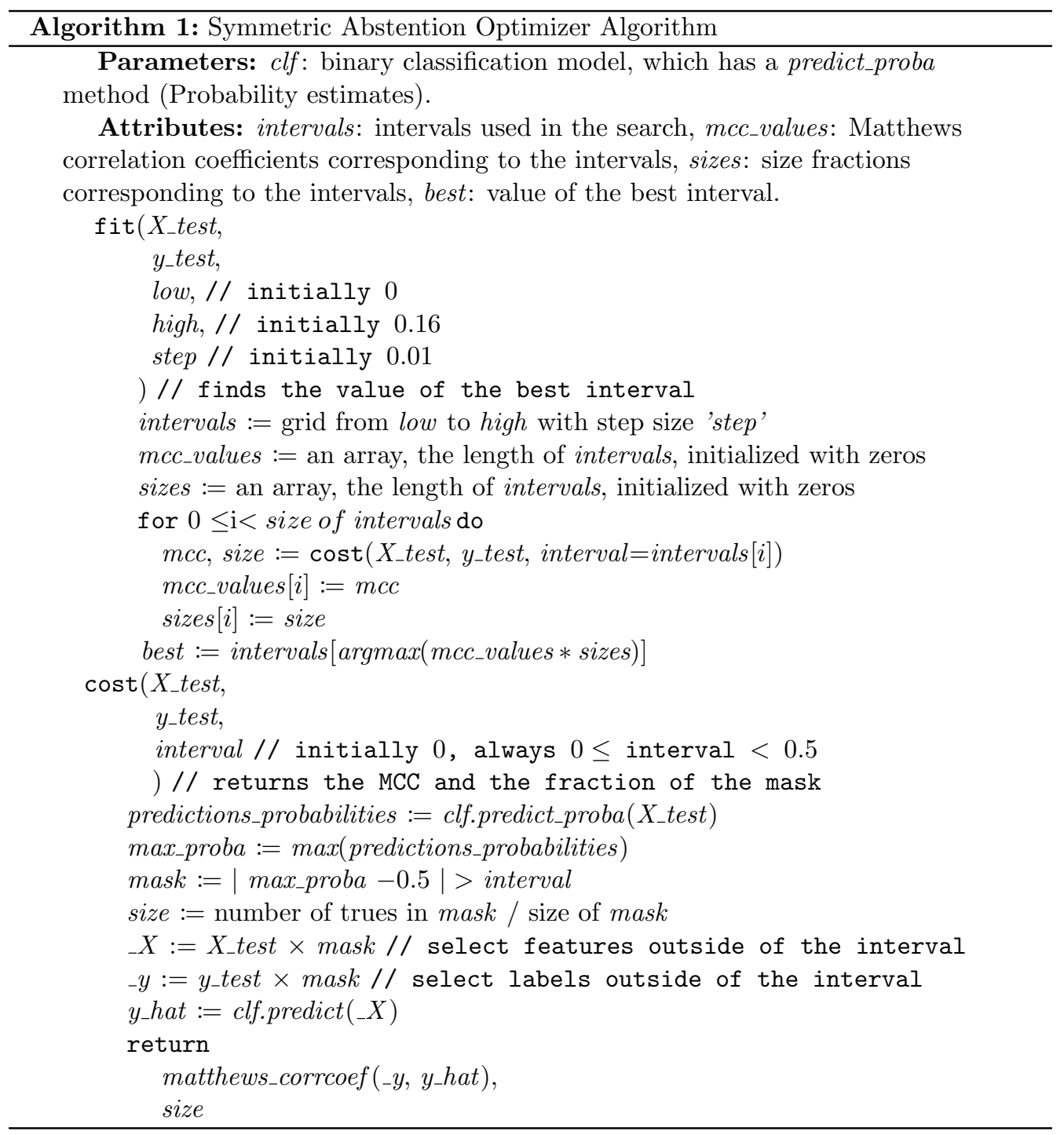



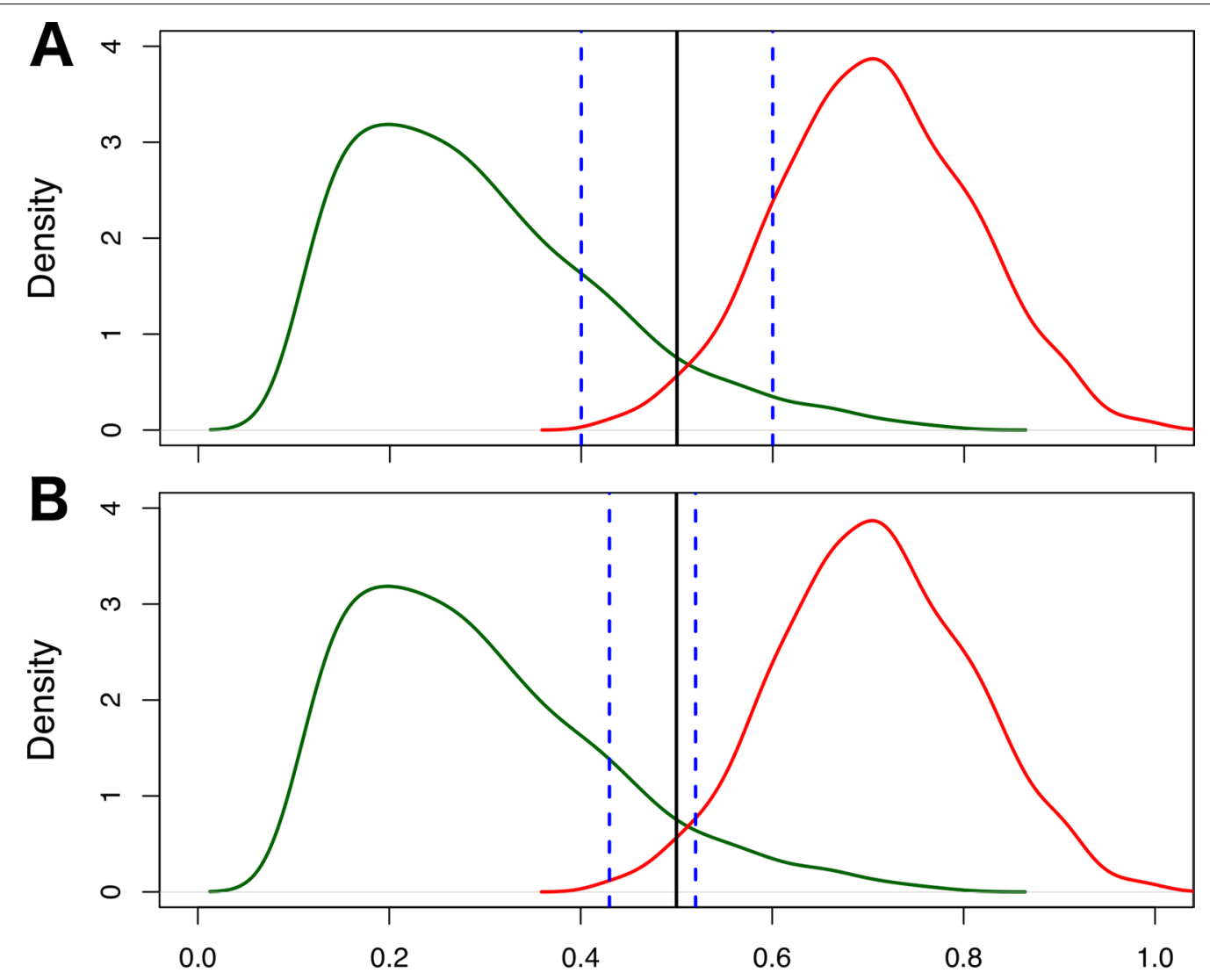

ML score

Fig. 1 Symmetric and asymmetric abstention. a Symmetric abstention; b asymmetric abstention. The green curve represent the ML scores/ probabilities of the controls (negative class), whereas the red curve represents the ML scores/probabilities of the cases (positive controls). The black vertical line marks the decision boundary and the blue dashed lines mark the borders of the abstention interval

\section{Asymmetric abstention}

In contrast to the symmetric abstention interval method, in the asymmetric abstention interval method (see Figure 1) we have to search over the two parameters, namely the interval and also the anchor (i.e., the offset). The latter is basically the height of the interval, i.e., the center of the abstention line. The anchor and the interval are independent. Thus, the asymmetric abstention interval method will output the best interval and the best anchor, that maximize the output of the objective function argmax $\{($ mcc_values * sizes $)\}$. This means the best asymmetric abstention interval is straightforward, simply requiring computing $[L, U]$ with $L=0.5+$ best anchor - best interval and $U=0.5+$ best anchor + best interval. The best anchor gets added to the center 0.5 .

Thus, in this case, we create two variable ranges: the intervals and the anchors. Then we create MCCs and sizes, except that these are matrices now instead of arrays. To find the best combination, we use a two-dimensional grid search. We define the grid of the intervals and anchors that we want to search through, test each combination of possible parameters and select the best one for our asymmetric abstention interval. This approximation may be intractable in general since there would be infinitely many combinations to test for a continuous scale. The solution is to define a grid. This grid defines for each hyperparameter which values should be tested. In our case, where the intervals and the anchors are tuned: we could give the intervals the values between $(0,0.16)$ and the anchors the values between $(-0.2,0.2)$. The hypothesis is that there is a specific combination of values of the different hyperparameters that will maximize the product of $M C C$ and the size of the samples classified. So at each crossing point, the grid search will see what the maximum of the product mcc_values and sizes at this point is. After checking all the grid points, we know precisely which combination of parameters is the best. For the 
asymmetric abstention optimizer algorithm, we need some modifications of the core functions.

The fit () function in the asymmetric abstention interval method takes two additional parameters, namely (width and num_anchors), as input. We set them as follows: width $=0.2$, and num_anchors $=20$. The anchors start from - width to width with num anchors steps. The cost () function in the asymmetric abstention interval method takes one additional parameter (anchor) as input, which is initialized with 0 . Furthermore, the suitable range that we chose in the asymmetric abstention interval method is maximum probability $-0.5+$ anchor $\}$. So we add the anchor to add the offset of the asymmetry (see algorithm 2).

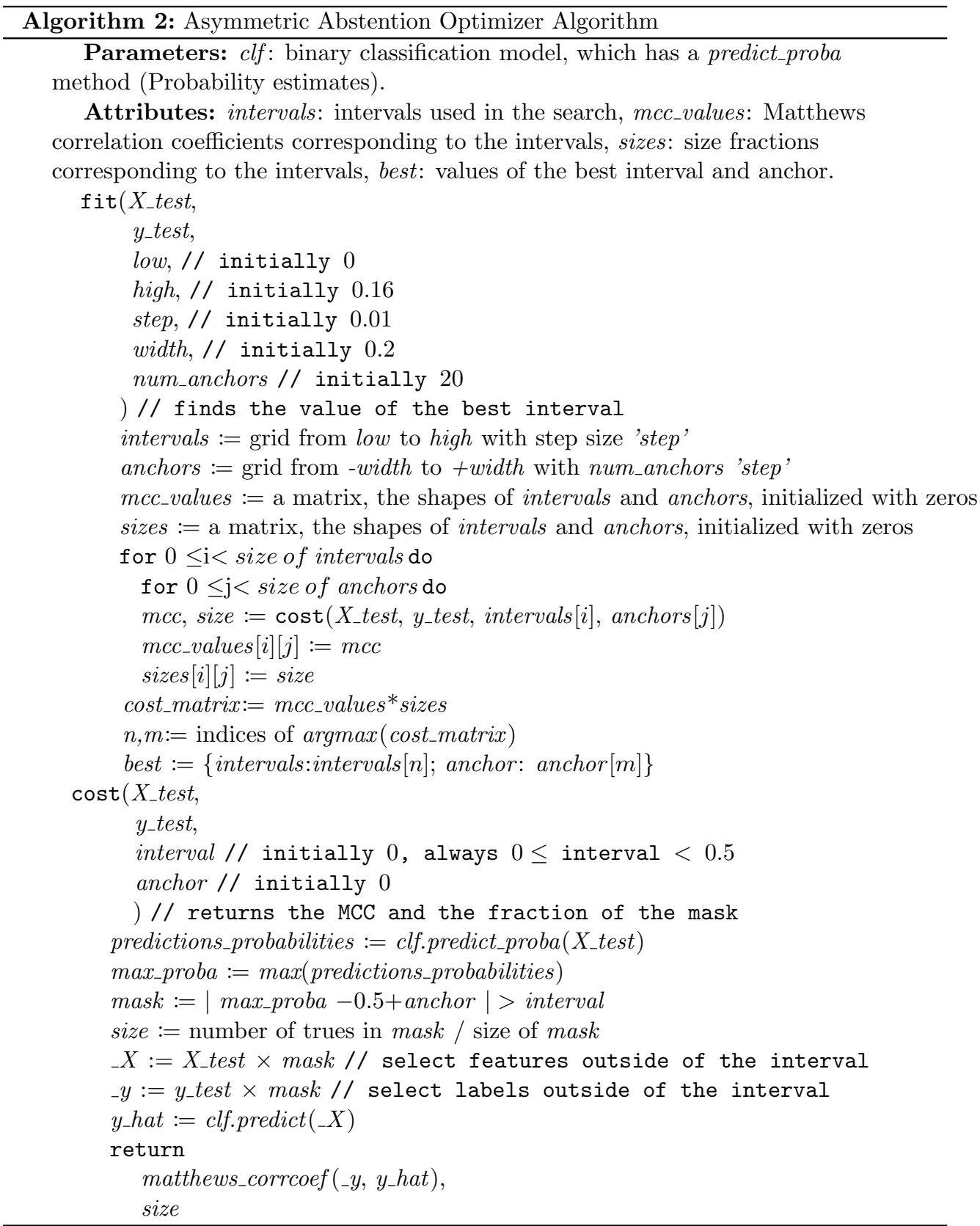




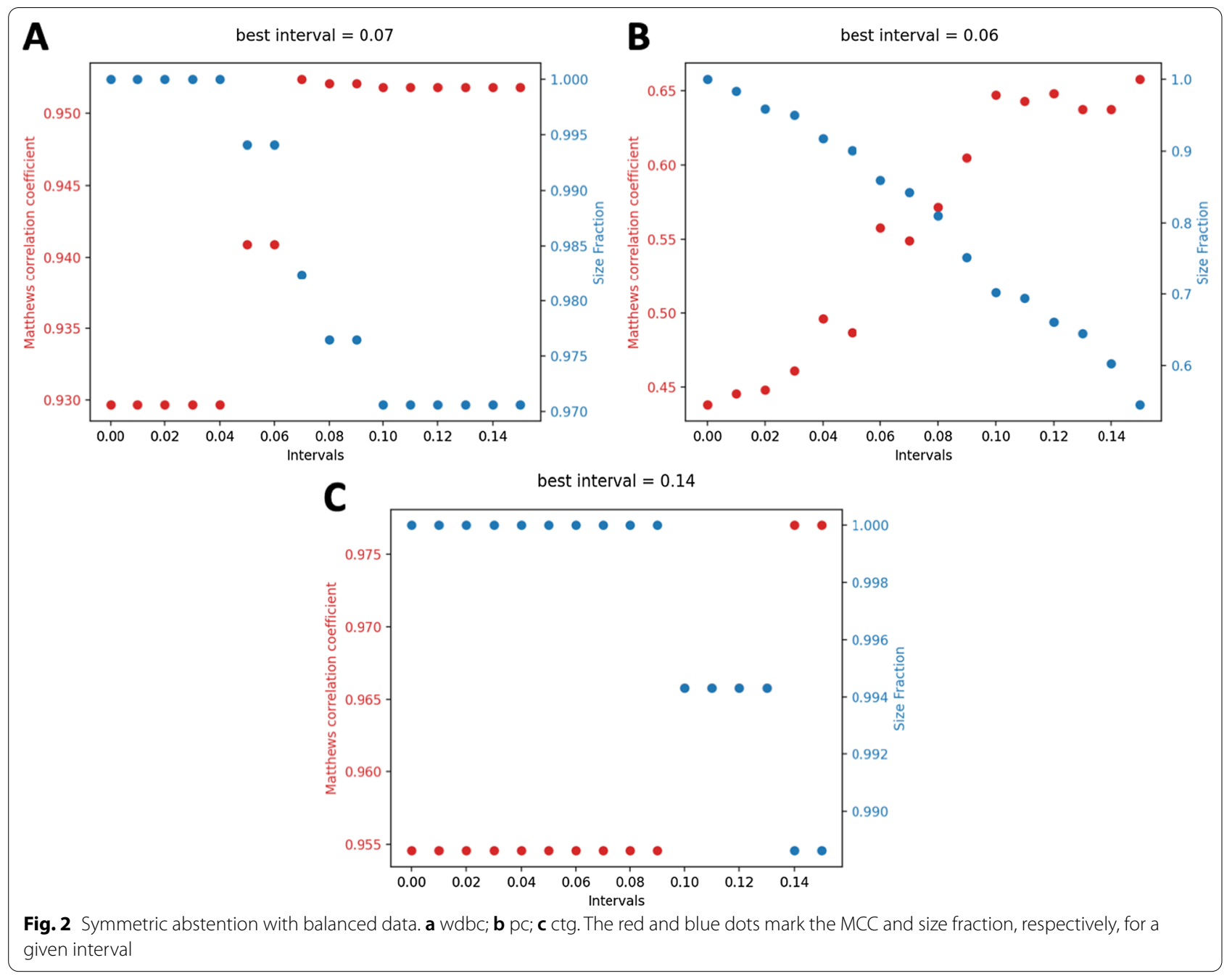

\section{Results}

We evaluated the symmetric and asymmetric abstention with three real-world datasets. To this end, we analyzed two scenarios, namely (1) the imbalanced data as it is and (2) down-sampled, balanced data.

For the wdbc dataset, the LR model performed well on the imbalanced data with an MCC of 0.925. For the balanced design, the LR model produced a similar performance with an MCC of 0.93. For the pc dataset, the LR model reached an MCC of 0.483 on the imbalanced data and an MCC of 0.438 on the balanced data. For the ctg dataset, the LR model achieved very high MCC values, namely 0.963 for the imbalanced and 0.955 for the balanced design.

The corresponding confusion matrices and the probability distributions of the controls and cases for all three datasets and the two evaluated scenarios (imbalanced and balanced design) can be found in Additional file 1: Figs. S1-S6.
We next evaluated and compared the performance of the symmetric and asymmetric abstention. The symmetric abstention performs well on balanced data and can significantly increase the MCC for all datasets. For instance, for the wdbc dataset, the best interval is [0.43, 0.57] with an MCC of 0.952 and a size fraction of $98.2 \%$ (see Figure 2A). For the pc dataset, the symmetric abstention on the balanced data performed best for an interval $[0.44,0.56]$ with an MCC of 0.558 and a size fraction of $86 \%$ (see Figure 2B). For the ctg dataset, the symmetric abstention was best with an interval [0.36, 0.64], resulting in an MCC of 0.977 and a size fraction of $98.9 \%$ (see Fig. 2c).

On imbalanced data, the symmetric abstention reaches an MCC of $0.951,0.556$, and 0.975 with corresponding size fractions of $97.4 \%, 87.4 \%$, and $99.6 \%$ for the datasets wdbc, pc, and ctg, respectively. The symmetric abstention can improve the MCC significantly. However, this comes with a rejection rate of up to $12.6 \%$. 


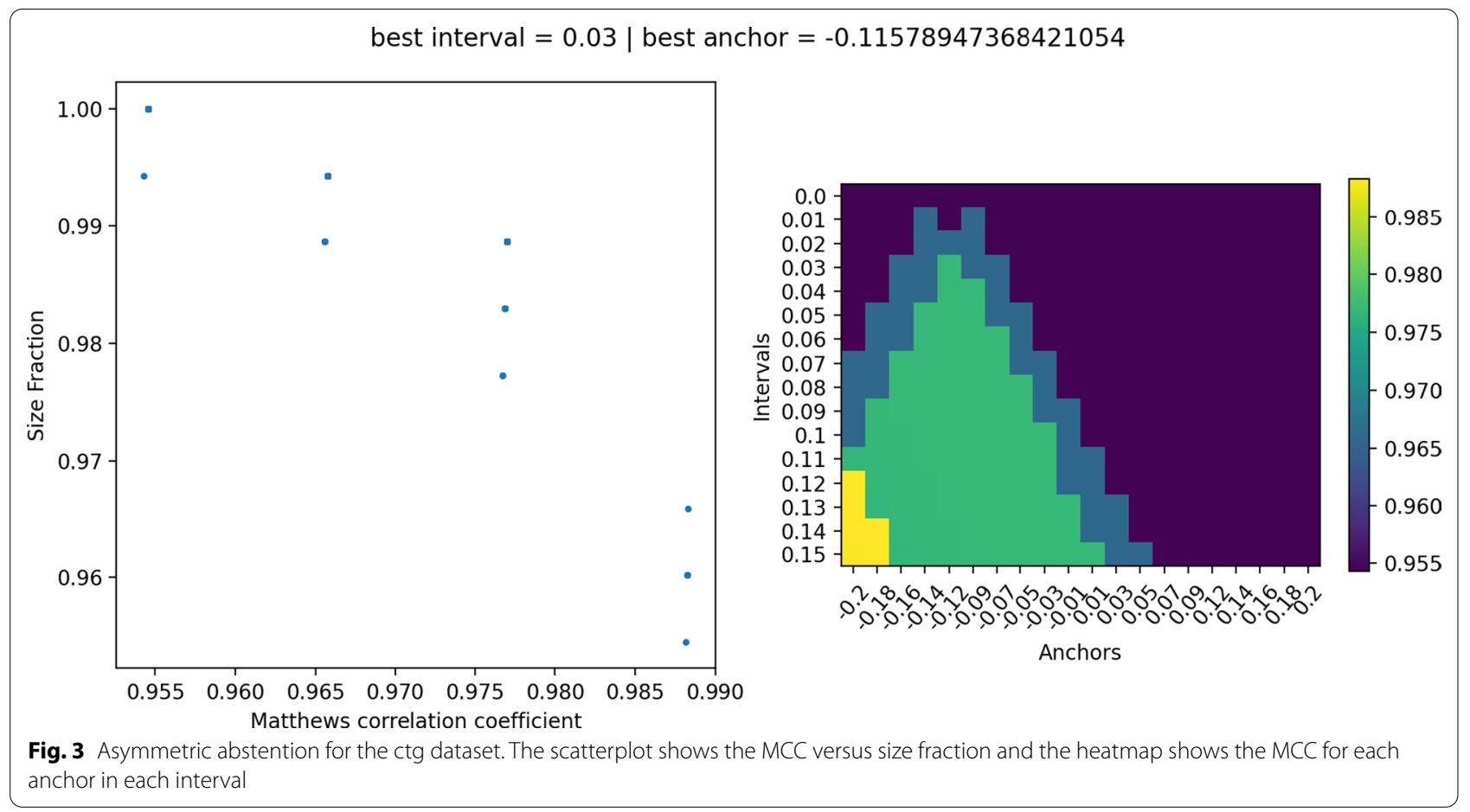

Table 5 Results of the models on the imbalanced data

\begin{tabular}{lllll}
\hline Name & Abstention & MCC & Rejected & Interval \\
\hline wdbc & None & 0.925 & - & - \\
wdbc & Symmetric & 0.951 & $<5 \%$ & {$[0.37,0.63]$} \\
wdbc & Asymmetric & 0.98 & $<5 \%$ & {$[0.263,0.463]$} \\
pc & None & 0.483 & - & - \\
pc & Symmetric & 0.556 & $<10 \%$ & {$[0.44,0.56]$} \\
pc & Asymmetric & 0.54 & $<10 \%$ & {$[0.427,0.467]$} \\
$c t g$ & None & 0.963 & - & - \\
ctg & Symmetric & 0.975 & $<1 \%$ & {$[0.39,0.61]$} \\
ctg & Asymmetric & 0.987 & $<1 \%$ & {$[0.252,0.432]$} \\
\hline
\end{tabular}

Abstention represents the abstention approach, i.e., none, symmetric, or asymmetric abstention. Rejected refers to the percentage of rejected samples, and Interval represents the bounderies of the abstention interval
Table 6 Results of the models on the balanced data

\begin{tabular}{lllll}
\hline Name & Abstention & MCC & Rejected & Interval \\
\hline wdbc & None & 0.93 & - & - \\
wdbc & Symmetric & 0.952 & $<5 \%$ & {$[0.43,0.57]$} \\
wdbc & Asymmetric & 0.952 & $<5 \%$ & {$[0.427,0.467]$} \\
pc & None & 0.438 & - & - \\
pc & Symmetric & 0.558 & $<10 \%$ & {$[0.44,0.56]$} \\
pc & Asymmetric & 0.558 & $<10 \%$ & {$[0.439,0.539]$} \\
ctg & None & 0.955 & - & - \\
ctg & Symmetric & 0.977 & $<5 \%$ & {$[0.36,0.64]$} \\
ctg & Asymmetric & 0.955 & $<1 \%$ & {$[0.354,0.414]$} \\
\hline
\end{tabular}

Abstention represents the abstention approach, i.e., none, symmetric, or asymmetric abstention. Rejected refers to the percentage of rejected samples, and interval represents the bounderies of the abstention interval
In contrast, the asymmetric abstention performs equally well in MCC for all imbalanced datasets, namely $0.98,0.54$, and 0.987 for the datasets wdbc, pc, and ctg, respectively. The rejection rate is similar to the rejection rate of the symmetric abstention approach with $4.3 \%, 7 \%$, and $0.8 \%$. However, it is always lower than $10 \%$, which is not the case for symmetric abstention. In Figure 3, the asymmetric abstention is exemplarily shown for the ctg dataset. The corresponding figures for the wdbc and pc datasets can be found in the Additional file 1: Figs. S7 and S8, respectively. All results of the imbalanced and balanced analyses are summarized in Tables 5 and 6.

\section{Discussion}

Our study demonstrates that both the symmetric and asymmetric abstention can improve the MCC for realworld classification, thereby improving the diagnostic value of an AI model in medical applications. However, this does not come without costs. In order to improve the MCC, the samples are rejected, which is particularly significant for the symmetric abstention on imbalanced data. In our study, we analyzed different datasets with different degrees of imbalance from moderate to high imbalance. Asymmetric abstention is particularly useful and superior for imbalanced data compared to 
symmetric abstention. Thus, asymmetric abstention should be considered when the dataset is imbalanced, which is regularly the case in medical datasets. Moreover, abstention is particularly useful for machine learning in automated processes to reduce costs for healthcare, in particular time and costs for medical staff. Thus, abstention in healthcare can be used for instance in screening processes to reduce the number of diagnoses with a human expert in the loop. In the future, we will extend our approach to general multi-class problems (i.e., classification tasks with more than two classes) with a reject option. Although it is just an extension of binary classification, it is more challenging for the algorithms to be effective. The more classes to predict, the more complex the problem will be. Our results show the usefulness and applicability of asymmetric abstention. However, there is room for improvements since our method does not solve all the problems associated with medical diagnostic decisions based on test scores [18], and we do not suggest that it replaces the use of the symmetric abstention interval method in general. In the future, we intend to analyze the interplay between data augmentation and abstention as well as the interplay between calibration methods for probabilistic interpretation and abstention intervals. Calibration can be used to make machine learning scores probabilistically interpretable and thus transform the original score distribution into a probability distribution, which directly affects the abstention interval. Moreover, we did not address at all the relevant regulatory requirements in our approach to be considered as software as a medical device (SAMD) [13, 25]. However, our new method can be a good starting point to improve diagnostic software for biomedical decision-making.

\section{Supplementary Information}

The online version contains supplementary material available at https://doi. org/10.1186/s12911-021-01655-y.

Additional file 1. Supplementary Figures 1-8.

\section{Authors' contributions}

$\mathrm{DH}$ designed and supervised the study. MG implemented the algorithms and performed the experiments. $\mathrm{DH}, \mathrm{MG}$, and $\mathrm{HH}$ interpreted the results. $\mathrm{DH}$ and MG wrote the manuscript. All authors read and approved the final manuscript.

\section{Funding}

Open Access funding enabled and organized by Projekt DEAL. This work is financially supported by the German Federal Ministry of Education and Research (BMBF) under the grant number 031L0267A (Deep-Insight).

\section{Availability of data and materials}

All datasets are publicly available. The Breast Cancer diagnostics dataset (wdbc) can be found at https://archive.ics.uci.edu/ml/datasets/Breast+Cancer+Wisconsin+\%28Diagnostic\%29, the Prostate Cancer (pc) dataset can be found in the R package lbreg https://cran.r-project.org/web/packages/lbreg/, and the Cardiotocography dataset (ctg) can be found at https://archive.ics.uci. edu/ml/datasets/Cardiotocography.

\section{Declarations}

Ethics approval and consent to participate

Not applicable.

\section{Consent for publication}

Not applicable.

\section{Competing interests}

The authors declare that they have no competing interests.

\section{Author details}

${ }^{1}$ Department of Data Science in Biomedicine, Faculty of Mathematics and Computer Science, University of Marburg, 35032 Marburg, Germany. ${ }^{2}$ Department of Statistics, Faculty of Mathematics and Computer Science, University of Marburg, 35032 Marburg, Germany.

Received: 11 August 2021 Accepted: 13 October 2021

Published online: 26 October 2021

\section{References}

1. Alvarsson J, McShane SA, Norinder U, Spjuth O. Predicting with confidence: using conformal prediction in drug discovery. J Pharm Sci. 2021;110:42-9. https://doi.org/10.1016/..xphs.2020.09.055.

2. Ayres de Campos D, Bernardes J, Garrido A, de sá JM, Pereira-leite L. Sisporto 2.0: a program for automated analysis of cardiotocograms. J Matern Fetal Med. 2000;5:311-8. https://doi.org/10.3109/14767050009053454.

3. Bartlett PL, Wegkamp MH. Classification with a reject option using a hinge loss. J Mach Learn Res (JMLR). 2008;9:1823-40.

4. Bibault J-E, Giraud P, Burgun A. Big data and machine learning in radiation oncology: state of the art and future prospects. Cancer Lett. 2016;382(1):110-7. https://doi.org/10.1016/j.canlet.2016.05.033.

5. Campagner A, Cabitza F, Ciucci D. The three-way-in and three-way-out framework to treat and exploit ambiguity in data. Int J Approx Reason. 2020;119:292-312. https://doi.org/10.1016/j.ijar.2020.01.010.

6. Chen P, Pan C. Diabetes classification model based on boosting algorithms. BMC Bioinform. 2018;19:109. https://doi.org/10.1186/ s12859-018-2090-9.

7. Chicco D, Jurman G. The advantages of the Matthews correlation coefficient (MCC) over f1 score and accuracy in binary classification evaluation. BMC Genomics. 2020;21:6.

8. Chow C. An optimum character recognition system using decision functions. IRE Trans Electron Comput. 1957:EC-6:247-54.

9. Chow C. On optimum recognition error and reject tradeoff. IEEE Trans Inf Theory. 1970;16:41-6. https://doi.org/10.1109/tit.1970.1054406.

10. Coudray N, Ocampo PS, Sakellaropoulos T, Narula N, Snuderl M, Fenyö D, Moreira AL, Razavian N, Tsirigos A. Classification and mutation prediction from non-small cell lung cancer histopathology images using deep learning. Nat Med. 2018;24:1559-67. https://doi.org/10.1038/ s41591-018-0177-5.

11. Dua D, Graff C. UCI machine learning repository (2017). http://archive.ics. uci.edu/ml.

12. Fumera G, Roli F, Giacinto G. Reject option with multiple thresholds. Pattern Recogn. 2000;33:2099-101. https://doi.org/10.1016/s0031-3203(00) 00059-5.

13. Hauschild A-C, Eick L, Wienbeck J, Heider D. Fostering reproducibility, reusability, and technology transfer in health informatics. Science. 2021;24(7):102803-1.

14. Heider D, Dybowski JN, Wilms C, Hoffmann D. A simple structure-based model for the prediction of HIV-1 co-receptor tropism. BioData Min. 2014. https://doi.org/10.1186/1756-0381-7-14.

15. Herbei R, Wegkamp MH. Classification with reject option. Can J Stat. 2006;34(4):709-21. https://doi.org/10.1002/cjs.5550340410.

16. Hernandez-Boussard T, Bozkurt S, loannidis JPA, Shah NH. MINIMAR (MINimum information for medical Al reporting): developing reporting standards for artificial intelligence in health care. J Am Med Inform Assoc. 2020;27(12):2011-5 
17. Hosmer DW, Lemeshow S. Applied logistic regression. New York: Wiley; 2000.

18. Landsheer JA. The clinical relevance of methods for handling inconclusive medical test results: quantification of uncertainty in medical decision-making and screening. Diagnostics. 2018;8(2):325. https://doi. org/10.3390/diagnostics8020032.

19. Lengauer T, Sing T. Bioinformatics-assisted anti-HIV therapy. Nat Rev Microb. 2006;4:790-7. https://doi.org/10.1038/nrmicro1477.

20. Libbrecht MW, Noble WS. Machine learning applications in genetics and genomics. Nat Rev Genet. 2015;16(6):321-32.

21. Madabhushi A, Lee G. Image analysis and machine learning in digital pathology: challenges and opportunities. Med Image Anal. 2016;33:1705. https://doi.org/10.1016/..media.2016.06.037.

22. Mortier T, Wydmuch M, Dembczynski K, Hüllermeier E, Waegeman W. Efficient set-valued prediction in multi-class classification. Data Min Knowl Discov. 2021;35(4):1435-69. https://doi.org/10.1007/s10618-021-00751-x.

23. Neumann U, Riemenschneider M, Sowa J-P, Baars T, Kälsch J, Canbay A, Heider D. Compensation of feature selection biases accompanied with improved predictive performance for binary classification by using a novel ensemble feature selection approach. BioData Min. 2016;9:36. https://doi.org/10.1186/s13040-016-0114-4.

24. Nguyen V-L, Destercke S, Masson M-H, Hülermeier E. Reliable multi-class classification based on pairwise epistemic and aleatoric uncertainty. In: Proceedings of the twenty-seventh international joint conference on artificial intelligence (IJCAI-18); 2018. p. 5089-95. https://doi.org/10.24963/ ijcai.2018/706

25. Riemenschneider M, Wienbeck J, Scherag A, Heider D. Data science for molecular diagnostics applications: from academia to clinic to industry. Syst Med. 2018;1:13-7. https://doi.org/10.1089/sysm.2018.0002.

26. Schwarz J, Heider D. Guess: projecting machine learning scores to wellcalibrated probability estimates for clinical decision making. Bioinformatics. 2019;35:2458-65.

27. Spänig S, Emberger-Klein A, Sowa J-P, Canbay A, Menrad K, Heider D. The virtual doctor: an interactive clinical-decision-support system based on deep learning for non-invasive prediction of diabetes. Artif Intell Med. 2019;100:101706. https://doi.org/10.1016/j.artmed.2019.101706.
28. Spänig S, Heider D. Encodings and models for antimicrobial peptide classification for multi-resistant pathogens. BioData Min. 2019;12:7. https:// doi.org/10.1186/s13040-019-0196-X.

29. Stekhoven DJ, Bühlmann P. Missforest—nonparametric missing value imputation for mixed-type data. Bioinformatics. 2012;28(1):112-8. https:// doi.org/10.1093/bioinformatics/btr597.

30. Tortorella F. An optimal reject rule for binary classifiers. In: Joint IAPR international workshops on statistical techniques in pattern recognition (SPR) and structural and syntactic pattern recognition (SSPR); 2000. p. 611-20. https://doi.org/10.1007/3-540-44522-6_63.

31. Tortorella F. Reducing the classification cost of support vector classifiers through an roc-based reject rule. Pattern Anal Appl. 2004;7(2):128-43. https://doi.org/10.1007/s10044-004-0209-2.

32. Tsybakov AB. Optimal aggregation of classifiers in statistical learning. Ann Stat. 2004;32(1):135-66. https://doi.org/10.1214/aos/1079120131.

33. Wolberg WH, Mangasarian OL. Multisurface method of pattern separation for medical diagnosis applied to breast cytology. Proc Natl Acad Sci U S A. 1990;87(23):9193-6. https://doi.org/10.1073/pnas.87.23.9193.

34. Wu E, Wu K, Daneshjou R, Ouyang D, Ho DE, Zou J. How medical Al devices are evaluated: limitations and recommendations from an analysis of FDA approvals. Nat Med. 2021;27(4):582-4. https://doi.org/10.1038/ s41591-021-01312-x.

35. Yala A, Barzilay R, Salama L, Griffin M, Sollender G, Bardia A, Lehman C, Buckley JM, Coopey SB, Polubriaginof F, Garber JE, Smith BL, Gadd MA, Specht MC, Gudewicz TM, Guidi AJ, Taghian A, Hughes KS. Using machine learning to parse breast pathology reports. Breast Cancer Res Treat. 2017;161:203-11. https://doi.org/10.1007/s10549-016-4035-1.

\section{Publisher's Note}

Springer Nature remains neutral with regard to jurisdictional claims in published maps and institutional affiliations.
Ready to submit your research? Choose BMC and benefit from:

- fast, convenient online submission

- thorough peer review by experienced researchers in your field

- rapid publication on acceptance

- support for research data, including large and complex data types

- gold Open Access which fosters wider collaboration and increased citations

- maximum visibility for your research: over $100 \mathrm{M}$ website views per year

At BMC, research is always in progress.

Learn more biomedcentral.com/submissions 